\title{
How to Define the Units of the Revised SI Starting from Seven Constants with Fixed Numerical Values
}

\author{
Richard S. Davis \\ International Bureau of Weights and Measures \\ Sèvres, France \\ rdavis@bipm.org
}

\begin{abstract}
As part of a revision to the International System of Units (SI) approved in 2018 and to take effect in May 2019, the seven base units will be defined by giving fixed numerical values to seven defining constants. This article shows how the definitions of all seven base units can be derived efficiently from the defining constants, with the result appearing as a table. The table's form makes evident a number of connections between the defining constants and the base units. Appendices show how the same methodology could have been used to define the same base units in the present SI, as well as the mathematics that underpins the methodology. Since the base units are now defined in terms of constants, then all units in the SI are now defined in terms of those constants.
\end{abstract}

Key words: base units; defining constants; fundamental constants; International System of Units.

Accepted: November 16, 2018

Published: December 3, 2018

https://doi.org/10.6028/jres.123.021

\section{Introduction}

Preparations for the upcoming revision of the International System of Units (SI) began in earnest with Resolution 1 of the 24th meeting of the General Conference on Weights and Measures (CGPM) in 2011 [1]. The 26th CGPM in November 2018 gave its final approval to a revision of the present SI [2] based on the guidance that had already been laid down in Ref. [1]. The SI will consequently become a system of units based on exact numerical values of seven defining constants, $\Delta v_{\mathrm{Cs}}, c, h, e, k, N_{\mathrm{A}}$, and $K_{\mathrm{cd}}$ exactly as specified in the following bullet points:

- The unperturbed ground-state hyperfine transition frequency of the cesium-133 atom $\Delta v_{\mathrm{Cs}}$ is 9192631770 hertz.

- The speed of light in vacuum $c$ is 299792458 meters per second.

- The Planck constant $h$ is $6.62607015 \times 10^{-34}$ joule second.

- The elementary charge $e$ is $1.602176634 \times 10^{-19}$ coulomb.

- The Boltzmann constant $k$ is $1.380649 \times 10^{-23}$ joule per kelvin.

- The Avogadro constant $N_{\mathrm{A}}$ is $6.02214076 \times 10^{23}$ reciprocal mole.

- The luminous efficacy of monochromatic radiation of frequency $540 \times 10^{12} \mathrm{~Hz}, K_{\text {cd }}$, is 683 lumens per watt. 
The hertz, joule, coulomb, lumen, and watt, with unit symbols Hz, J, C, lm and W, respectively, are related to the seven base units: second, meter, kilogram, ampere, kelvin, mole, and candela, with unit symbols $\mathrm{s}, \mathrm{m}, \mathrm{kg}, \mathrm{A}, \mathrm{K}, \mathrm{mol}$, and $\mathrm{cd}$, respectively, through the relations $\mathrm{Hz}=\mathrm{s}^{-1}, \mathrm{~J}=\mathrm{kg} \mathrm{m}^{2} \mathrm{~s}^{-2}, \mathrm{C}=\mathrm{A} \mathrm{s}$, $\mathrm{lm}=\mathrm{cd} \mathrm{sr}$, and $\mathrm{W}=\mathrm{kg} \mathrm{m}^{2} \mathrm{~s}^{-3}$ [2]. ${ }^{1}$ Only "coherent" units [2] are used here; just as in the bullet points, we will not affix numerical prefixes (such as mega or nano) either to the base units or to combinations of units that have special names (such as joule or watt).

The numerical values of $\Delta v_{\mathrm{Cs}}, c$, and $K_{\mathrm{cd}}$ given in the bullet points have been fixed (defined to be exact) since 1967, 1983, and 1979 respectively [2]. It was premature in 2011 to specify exact values for $h$, $e, k$, and $N_{\mathrm{A}}$ because their experimentally determined values were not yet known with sufficiently small uncertainty to assure a smooth transition to the proposed new definitions. That has changed, and the numerical values given above are those recommended in October 2017 by the CODATA Task Group on Fundamental Constants [3]. They have been accepted by the International Committee for Weights and Measures (CIPM) and were confirmed by the CGPM at its meeting in November 2018.

This article describes an efficient method to convert the information contained in the seven bullet points to definitions of the SI base units, which are, not coincidentally, seven in number [2]. The reasons for this particular choice of defining constants are important but have been presented elsewhere [4].

Starting with the seven bullet points, we derive in one step the combinations of the seven defining constants that define the base units of the SI. The algorithm results in an easy-to-read table. The exact numerical values given in the bullet points are then introduced to complete the definitions of the base units. Appendix A applies the same method to the present SI, illustrating the method's generality as well as providing a novel contrast to the revision. Each base unit will be defined without reference to any of the others. Appendix B describes a more abstract method of defining the base units from the values of the defining constants and compares the abstract formalism with that presented in the two sections below.

If all seven base units of the SI can be defined in terms of the seven defining constants, an obvious but important corollary follows: All SI units can be defined in terms of the seven defining constants.

The distinction between base and derived units remains useful, but not essential for many purposes. ${ }^{2}$

The following method is consistent with a more rigorous analysis provided by Mohr in 2008 [6], which the interested reader is encouraged to consult.

\section{Defining Constants Written in Terms of Base Units}

We begin with Table 1, which presents much of the information given in the bullet points of Section 1 in more usable form. Note that the defining constants are shown as labels in the first column. The four new defining constants and the base units which are redefined in consequence are shown in red. The units of the defining constants can be expressed as the product of powers of the base units, $\mathrm{s}^{\alpha} \mathrm{m}^{\beta} \mathrm{kg}^{\gamma} \mathrm{A}^{\delta} \mathrm{K}^{\varepsilon} \mathrm{mol}$ $\operatorname{cd}^{\eta}[2]$, as specified in the bullet points of Section 1. The required exponents appear in the rows of Table 1 for each of the defining constants; for example, the coherent unit of the Planck constant $h$ is $\mathrm{J} \mathrm{s}=\mathrm{kg} \mathrm{m}^{2} \mathrm{~s}^{-1}$, so that for the row labeled $h$ the exponents $\alpha$ through $\eta$ are $(-1,2,1,0,0,0,0)$. The columns show whether a unit appears in a particular bullet point. We see, for instance, that the second appears in the unit of every constant except $N_{\mathrm{A}}$, but with four different exponents.

\footnotetext{
${ }^{1} \mathrm{sr}$ is the symbol for steradian, the unit of solid angle. Although $\mathrm{sr}=\mathrm{m}^{2} / \mathrm{m}^{2}=1$, $\mathrm{sr}$ is used when needed for clarity [2].

${ }^{2}$ Even early editions of the SI Brochure remarked that separate classes of base and derived units are "not essential to the physics of the subject" [5], but added that the classifications were useful, considering the goal of "a single, practical, worldwide system [of units] for international relations, for teaching and scientific work."
} 
Table 1. Units of the defining constants in terms of powers of the base units.

\begin{tabular}{|c|c|c|c|c|c|c|c|}
\cline { 2 - 8 } \multicolumn{1}{c|}{} & $\mathbf{s}$ & $\mathbf{m}$ & $\mathbf{k g}$ & $\mathbf{A}$ & $\mathbf{K}$ & $\mathbf{m o l}$ & $\mathbf{c d}$ \\
\hline $\boldsymbol{\Delta} \boldsymbol{v} \mathbf{C s}$ & -1 & 0 & 0 & 0 & 0 & 0 & 0 \\
\hline $\boldsymbol{c}$ & -1 & 1 & 0 & 0 & 0 & 0 & 0 \\
\hline $\boldsymbol{h}$ & -1 & 2 & 1 & 0 & 0 & 0 & 0 \\
\hline $\boldsymbol{e}$ & 1 & 0 & 0 & 1 & 0 & 0 & 0 \\
\hline $\boldsymbol{k}$ & -2 & 2 & 1 & 0 & -1 & 0 & 0 \\
\hline $\boldsymbol{N}_{\mathbf{A}}$ & 0 & 0 & 0 & 0 & 0 & -1 & 0 \\
\hline $\boldsymbol{K}_{\mathbf{c d}}$ & 3 & -2 & -1 & 0 & 0 & 0 & 1 \\
\hline
\end{tabular}

The sequence of the seven defining constants in the left column of labels follows the order in which they are presented in the CGPM Resolution and in Section 1. The sequence of base units in the top row of labels follows the order in which these units are defined in Ref. [4]. This results in a table where the exponents above the diagonal cells are all zero (as are most exponents below the diagonal). The diagonal cells (those with a violet background) associate each defining constant with a "primary" base unit. The cells with a yellow background show that "helping" units are also needed. For instance, the Planck constant $h$ is key to redefining the kilogram (violet cell), but the unit of $h$ also contains the inverse second and the meter squared (yellow cells).

\section{Base Units as Defined by the "Defining Constants"}

The seven SI base units can be defined in terms of the seven defining constants. To do this, we create a second table, Table 2 , that shows the combination of defining constants required to define each base unit. Table 2 is the major contribution of this report.

The numbers in Table 2 are also exponents, this time used to show the combination of defining constants (labeled in the top row) that has the same unit as each base unit (labeled in the left column). Except for an exact scaling factor, which is easily derived as shown below, each base unit is defined by the product $\Delta v_{\mathrm{Cs}}{ }^{\alpha} c^{\beta^{\prime}} h^{\gamma^{\prime}} e^{\delta^{\prime}} k^{\varepsilon^{\prime}} N_{\mathrm{A}}^{\zeta} K_{\mathrm{cd}}{ }^{\eta^{\prime}}$, where the required exponents for each row appear in the table. If an exponent is zero, it means that its constant is not needed, and its cell, though containing zero, has been left blank for visual clarity. Each column shows which defining constants are needed in the definition of the base units. We see that $\Delta v_{\mathrm{Cs}}$ is needed to define six of the seven base units (using three different exponents), $c$ is only needed to define two base units, etc.

All exponents have been derived from Table 1 in one step using the following mathematical operation. Note that the cells containing numbers in Table 1 constitute a $7 \times 7$ matrix. Invert that matrix using, for example, the MINVERSE command in Excel. The inverse obtained is the $7 \times 7$ matrix of exponents shown in Table $2 .{ }^{3}$ Remember that the blank cells actually contain zero.

From the "kg" row of Table 2 we may infer that the following combination of three defining constants has the kilogram as its unit [6]:

$$
\Delta v_{\mathrm{Cs}}^{1} c^{-2} h^{1} e^{0} k^{0} N_{\mathrm{A}}^{0} K_{\mathrm{cd}}^{0}=\Delta v_{\mathrm{Cs}}^{1} c^{-2} h^{1}=\frac{\Delta v_{\mathrm{Cs}} h}{c^{2}}
$$

\footnotetext{
${ }^{3}$ The $7 \times 7$ squares of numbers in Tables 1 and 2 are called "lower triangular matrices" because all numbers above the diagonal are zero. The inverse of a triangular matrix is triangular as well, provided that the inverse exists. The inverse exists if and only if none of the numbers in the diagonal cells is zero.
} 


\section{Journal of Research of the National Institute of Standards and Technology}

Table 2. The revised SI [4]. Blank cells all contain zero (not displayed).

\begin{tabular}{|c|c|c|c|c|c|c|c|}
\cline { 2 - 8 } \multicolumn{1}{c|}{} & $\boldsymbol{\Delta} \boldsymbol{v} \mathbf{C s}$ & $\boldsymbol{c}$ & $\boldsymbol{h}$ & $\boldsymbol{e}$ & $\boldsymbol{k}$ & $\boldsymbol{N}_{\mathrm{A}}$ & $\boldsymbol{K}_{\mathbf{c d}}$ \\
\hline $\mathbf{s}$ & -1 & & & & & & \\
\hline $\mathbf{m}$ & -1 & 1 & & & & & \\
\hline $\mathbf{k g}$ & 1 & -2 & 1 & & & & \\
\hline $\mathbf{A}$ & 1 & & & 1 & & & \\
\hline $\mathbf{K}$ & 1 & & 1 & & -1 & & \\
\hline $\mathbf{m o l}$ & & & & & & -1 & \\
\hline $\mathbf{c d}$ & 2 & & 1 & & & & 1 \\
\hline
\end{tabular}

In the revised SI, all mass determinations must ultimately be traceable to this quantity because its numerical value in kilograms has been fixed. The exact values of $\Delta v_{\mathrm{Cs}}, c$ and $h$ given in the bullet points of Section 1 provide the fixed value. Substituting the information in the first three bullet points into the left side of the following equation, which is a combination of physical constants, gives us the right side, which is the value of the combination in the revised SI:

$$
\frac{\Delta v_{\mathrm{Cs}} h}{c^{2}}=\frac{(9192631770)\left(6.62607015 \times 10^{-34}\right)}{(299792458)^{2}} \mathrm{~kg}
$$

The numbers in parentheses are obviously the exact numerical values of $\Delta v_{\mathrm{Cs}}, c$, and $h$ specified in Section 1 . The base units associated with these numerical values cancel (unit symbols can be treated algebraically)—except for the kilogram! Then, by simple arithmetic,

$$
1 \mathrm{~kg}=\frac{(299792458)^{2}}{(9192631770)\left(6.62607015 \times 10^{-34}\right)} \frac{\Delta v_{\mathrm{Cs}} h}{c^{2}}=1.4755213997 \ldots \times 10^{40} \frac{\Delta v_{\mathrm{Cs}} h}{c^{2}}
$$

Any given base unit can be defined similarly, without knowing the definitions of any other base units. Only the exact numerical values of the defining constants are required. This definition of the kilogram appears in Section 2.3.1 of Ref. [4]. A more formal derivation of the seven definitions, of which Eq. (2) is an example, is provided in Appendix B, along with a comparison to the simplified approach adopted above. Reference [7] reaches the same conclusions following a different path.

It is irrelevant that the mass $\Delta v_{\mathrm{Cs}} h / c^{2}$ is so miniscule that it must be scaled up by 40 orders of magnitude to equal one kilogram. It has always been true that "any method consistent with the laws of physics could be used to realize any SI unit" [2] and such methods already exist for the kilogram as it will be defined by Eq. (2) [4]. Appendix A discusses in more detail the condition that assures continuity of the redefined kilogram with the present kilogram.

\section{Summary and Discussion}

Several pictorial illustrations of the revised SI have been available [8], [9]. In one case, readers are cautioned that the illustration is not an explanation [8]. By contrast, Table 2 has been derived mathematically from the seven defining constants, knowing only their units. It is easily noted in Table 2 that: 
- The violet cells on the diagonal connect a base unit in the left column with the constant which defines it, in the top row. This is loose terminology because, in most instances, one or two "helping constants" are essential, and these are shown in the yellow cells of each row. All other cells contain zero, and these are left blank.

- There are only three helping constants, $\Delta v_{\mathrm{Cs}}, c$, and $h$, and these also serve as the defining constants for the second, meter and kilogram, respectively. [It is perhaps noteworthy that the second, meter, and kilogram are the mechanical units of the old meter-kilogram-second (MKS) system, from which the SI evolved.]

- In each row, the product of powers of the constants in the violet cell and any yellow cells form a quantity (which is also a constant) whose unit is the base unit of the row. The exponents needed are shown.

- At most, two helping constants are required to define any base unit. [The appearance of helping constants can be viewed as a mathematical requirement which reconciles continued use of the historical base units with the most useful selection of defining constants. See Appendix A, which shows that the present SI [2] is not very different in this respect.]

- Helping constants are not needed to define either the second or the mole.

- The ground state hyperfine transition frequency of the cesium-133 atom $\Delta v_{\mathrm{CS}}$ is needed in the definitions of all base units except the mole.

- The speed of light in vacuum $c$ is needed only in the definitions of the meter and kilogram.

- The Planck constant $h$ is needed only in the definitions of the kilogram, kelvin and candela.

- The elementary charge $e$, the Boltzmann constant $k$, the Avogadro constant $N_{\mathrm{A}}$ and the luminous efficacy of a specified wavelength $K_{\text {cd }}$ are each needed to define a single base unit (ampere, kelvin, mole, and candela respectively). They are not used as helping constants.

- The importance of the unit of time to the revised SI is evident.

- As emphasized in the Introduction, all SI units can be written in terms of the seven defining constants. As examples of this, the reader may wish to verify that the joule is defined in terms of $h \Delta v_{\mathrm{Cs}}$, and the ohm in terms of $h / e^{2}$.

There is no general requirement that exponents in Tables 1 and 2 must be displayed as lower triangular matrices (see Appendix B), although this arrangement makes the tables easier to scan visually and therefore has merit. Because Table 1 is a lower triangular matrix, one can see that the units can also be defined in seven separate steps rather than as a group, as we have done. The step-by-step method, also used in the draft 9th edition of the SI Brochure [4], first defines the SI second from the upper left corner of Table 1. The meter can then be defined from the next row because the helping unit of $c$, the second, has already been defined. The kilogram can be defined from the third row because the two helping units of $h$ have already been defined. All helping units have now been defined and so the remaining four SI units can be defined in any order one wishes, including of course the order found in [4].

The seven unique combinations of defining constants whose unit is a base unit [Eq. (1) shows the combination for the kilogram] were derived together by matrix inversion. Since any order of units and defining constants used as labels in Table 1 leads to identical definitions of the base units, we have chosen an order that makes Table 2 visually simple. It is also the order found in the major reference for the revised SI [4]. 


\section{Journal of Research of the National Institute of Standards and Technology}

\section{Appendix A: The SI Prior to May 20, 2019}

The SI in use at present [2] could also have been formulated in terms of the six defining constants and one defining quantity that had been the most-recently specified by the CGPM, either explicitly or implicitly, prior to November 2018 (see Ref. [10]):

- The unperturbed ground state hyperfine transition frequency of the cesium-133 atom $\Delta v_{\mathrm{Cs}}$ is 9192631770 hertz (1967).

- $\quad$ The speed of light in vacuum $c$ is 299792458 meters per second (1983).

- The mass of the international prototype of the kilogram $m_{\mathcal{K}}$ is 1 kilogram (1889).

- The permeability of vacuum $\mu_{0}$ is $4 \pi \times 10^{-7}$ newton per ampere squared $(1948,1954)$.

- The thermodynamic temperature of the triple point of water $T_{\mathrm{TPW}}$ is $273.16 \mathrm{kelvin}$ (1954).

- The molar mass of carbon- $12, M\left({ }^{12} \mathrm{C}\right)$, is 0.012 kilogram per mole (1971).

- The luminous efficacy of monochromatic radiation of frequency $540 \times 10^{12} \mathrm{~Hz}, K_{\mathrm{cd}}$, is 683 lumens per watt (1979).

The newton $\left(\right.$ symbol $\mathrm{N}$ ) is expressed in terms of base units as $\mathrm{N}=\mathrm{kg} \mathrm{m} \mathrm{s}^{-2}$ [2]. Expressions for the hertz, lumen, and watt in terms of base units are found in Section 1.

Carrying out the same procedure as described in Sections 2 and 3 for the revised SI, we start with Table 3, which contains an embedded $7 \times 7$ matrix. Again, this matrix is a table of exponents inferred from seven bullet points, but now they are the bullet points found in this Appendix. For ease of comparison with Tables 1 and 2, the order of units in the top row of Table 3 is chosen to be identical to that of Table 1 , and the order of quantities in the left column is chosen to produce a lower triangular matrix. The order of quantities shown is the only one that does this.

Table 3. Units of the defining quantities in terms of powers of the base units (for the SI described in [2].

\begin{tabular}{|c|c|c|c|c|c|c|c|}
\cline { 2 - 8 } \multicolumn{1}{c|}{} & $\mathbf{s}$ & $\mathbf{m}$ & $\mathbf{k g}$ & $\mathbf{A}$ & $\mathbf{K}$ & $\mathbf{m o l}$ & $\mathbf{c d}$ \\
\hline $\boldsymbol{\Delta} \boldsymbol{\nu} \mathbf{C s}$ & -1 & 0 & 0 & 0 & 0 & 0 & 0 \\
\hline $\boldsymbol{c}$ & -1 & 1 & 0 & 0 & 0 & 0 & 0 \\
\hline $\boldsymbol{m}_{\mathcal{K}}$ & 0 & 0 & 1 & 0 & 0 & 0 & 0 \\
\hline $\boldsymbol{\mu}_{\boldsymbol{0}}$ & -2 & 1 & 1 & -2 & 0 & 0 & 0 \\
\hline $\boldsymbol{T}_{\mathrm{TPW}}$ & 0 & 0 & 0 & 0 & 1 & 0 & 0 \\
\hline $\boldsymbol{M}^{\left({ }^{12} \mathbf{C}\right)}$ & 0 & 0 & 1 & 0 & 0 & -1 & 0 \\
\hline $\boldsymbol{K}_{\mathbf{c d}}$ & 3 & -2 & -1 & 0 & 0 & 0 & 1 \\
\hline
\end{tabular}

Now transpose the labels of Table 3 and invert its embedded matrix to arrive at Table 4. As with Table 2 , cells containing zero are left blank. 


\section{Journal of Research of the National Institute of Standards and Technology}

Table 4. The SI described in [2]. The blank cells all contain zero (not displayed).

\begin{tabular}{|c|c|c|c|c|c|c|c|}
\cline { 2 - 8 } \multicolumn{1}{c|}{} & $\boldsymbol{\Delta} \boldsymbol{\nu}_{\mathbf{C s}}$ & $\boldsymbol{c}$ & $\boldsymbol{m}_{\mathcal{K}}$ & $\boldsymbol{\mu}_{0}$ & $\boldsymbol{T}_{\mathrm{TPW}}$ & $\boldsymbol{M}\left({ }^{12} \mathbf{C}\right)$ & $\boldsymbol{K}_{\mathbf{c d}}$ \\
\hline $\mathbf{s}$ & -1 & & & & & & \\
\hline $\mathbf{m}$ & -1 & 1 & & & & & \\
\hline $\mathbf{k g}$ & & & 1 & & & & \\
\hline $\mathbf{A}$ & $1 / 2$ & $1 / 2$ & $1 / 2$ & $-1 / 2$ & & & \\
\hline $\mathbf{K}$ & & & & & 1 & & \\
\hline $\mathbf{m o l}$ & & & 1 & & & -1 & \\
\hline $\mathbf{c d}$ & 1 & 2 & 1 & & & & 1 \\
\hline
\end{tabular}

This table can easily be compared to Table 2 . Note that only the first two rows, those for the second and meter, are identical in the two tables.

There is no reason that the exponents must be integers, as this example illustrates. Thus, in the SI described in Ref. [2], the ampere is realized by traceability to the quantity $\left(\Delta \nu_{\mathrm{Cs}} c m_{\mathcal{K}} / \mu_{0}\right)^{1 / 2}$ which, according to the information in the first four bullet points, has an exact value of about $1.5 \times 10^{12} \mathrm{~A}$. Note that the SI unit of $\Delta v_{\mathrm{Cs}} c m_{K}$ is the newton and that of $\mu_{0}$ is the newton per ampere squared.

The exponents appearing in Table 4 and the exact numerical values of the six constants and one physical quantity listed in the bullet points of Appendix A would have been sufficient to define all base units in Ref. [2]. For example, a definition equivalent to the present definition of the ampere [2] would be:

$$
1 \mathrm{~A}=\left[\frac{4 \pi \times 10^{-7}}{(9192631770)(299792458)(1)}\right]^{1 / 2}\left(\frac{\Delta v_{\mathrm{Cs}} c m_{\mathcal{K}}}{\mu_{0}}\right)^{1 / 2}=6.752656 \ldots \times 10^{-13}\left(\frac{\Delta v_{\mathrm{Cs}} c m_{\mathcal{K}}}{\mu_{0}}\right)^{1 / 2} .
$$

In this adaptation of Ref. [2], $m_{K}$ is the sole defining quantity that is not some kind of constant. Rather, it is the mass of an artefact known as the International Prototype of the Kilogram, $\mathcal{K}$, which has been used since 1889 to define one kilogram [2]. This artefact definition of the kilogram is simple, understandable, and independent of the six constants. Unfortunately, since the mass of $\mathcal{K}$ is not a physical constant, the stability over time of the unit it defines cannot be assured. The same lack of assurance affects, at least in principle, the three units for which $m_{K}$ is a "helper," one of which is the ampere (see yellow cells in the column of Table 4 labeled " $m_{\mathcal{K}}$ "). When the SI was first approved by the 11 th CGPM in 1960, it was recognized that the artefact definition of the kilogram was a weakness of the International System of Units - to be remedied "sooner or later" [11].

The present definition of the kilogram [2] is contained entirely in the third bullet point of Appendix A. In symbols, $1 \mathrm{~kg}=m_{\mathcal{K}}$.

The revised definition of the kilogram [4] is given by Eq. (2),

$$
1 \mathrm{~kg}=1.4755213997 \ldots \times 10^{40} \frac{\Delta v_{\mathrm{Cs}} h}{c^{2}}
$$

The value of the prefactor on the right side ensures that there will be no perceptible discontinuity in the kilogram unit when the redefinition comes into force [3], [4]. The continuity condition requires that the weighted mean of the most accurate experimental values of $h$ has become fixed [3] so that, just after the redefinition comes into force on 20 May 2019,

$$
x \cdot m_{\mathcal{K}}=1.4755213997 \ldots \times 10^{40} \frac{\Delta v_{\mathrm{Cs}} h}{c^{2}}
$$


where the experimental value of $x$ is unity to within a relative uncertainty that is sufficiently small $\left(1 \times 10^{-8}[3]\right)$ to make the redefinition imperceptible to the vast majority of users. (Subsequently, the experimental value of $x$ might change simply because $m_{\mathcal{K}}$ is not a physical constant. Time will tell.) The impact of the revised SI on most users of the present SI has been assessed to be small by international experts [12].

\section{Appendix B: Derivation of Definitions of the Base Units in the Revised SI ${ }^{4}$}

\subsection{Preliminary Consideration of the Defining Constants Specified in the Bullet Points of Section 1}

Let $C_{i}$ be the symbol for the $i^{\text {th }}$ defining constant and let its fixed numerical value be $N_{i}$ when expressed in the SI coherent unit $U_{i}$. Because $C_{i}$ is one of seven defining constants, the index $i$ runs from 1 to 7 . The set of $C_{i}$ is comprised of $\Delta v_{\mathrm{Cs}}, c, h, e, k, N_{\mathrm{A}}$, and $K_{\mathrm{cd}}$. The subscript $i$ assigned to the defining constants is an arbitrary choice. We have chosen here the order in which the bullet points are listed in Section 1.

The $i^{\text {th }}$ bullet point of Section 1 can be written in generic symbols as

$$
C_{i}=N_{i} \cdot U_{i}
$$

The defining constants are quantities that appear in the equations of physics. The right side of Eq. (B1) is the exact value of each $C_{i}$ in the revised SI because the numerical values $N_{i}$ have been chosen to be exact. The bullet points of Section 1 therefore define the coherent unit $U_{i}$ in terms of the quantity $C_{i}$, which is a constant of some type [4].

Any coherent unit of the SI can be expressed as the product of powers of the seven base units $[2,4]$. We refer below to the $i^{\text {th }}$ base unit as $B_{i}$. The symbols for the seven base units are: $\mathrm{s}, \mathrm{m}, \mathrm{kg}, \mathrm{A}, \mathrm{K}, \mathrm{mol}$, and cd. Because units are commutative, the index $i$ assigned to each base unit is also an arbitrary choice and need not follow the order shown here, although this is the order adopted in Sections 2 and 3 of this report and in Ref. [4]. Reference [2], for instance, adopts a different order. The SI unit $U_{i}$ of each defining constant is given in terms of the base units by

$$
U_{i}=\prod_{j=1}^{7} B_{j}^{a_{i j}} .
$$

The exponents $a_{i j}$ are easily inferred from the seven bullet points in Section 1 and the supplementary information written just below them. The exponents turn out to be integers ranging from -2 through +3 . Note that Eq. (B2) is merely a consequence of each defining constant being a quantity which has an SI unit.

\subsection{The Definitions of the Base Units}

Combining Eqs. (B1) and (B2) to eliminate $U_{i}$, we obtain

$$
\frac{C_{i}}{N_{i}}=\prod_{j=1}^{7} B_{j}^{a_{i j}}
$$

The task now is to express any given base unit $B_{i}$ as the product of powers of the seven ratios $C_{j} / N_{j}$. We therefore seek the "inverse form" of Eq. (B3).

Temporarily treating the symbols of quantities and units as algebraic abstractions, we take the logarithm ${ }^{5}$ of both sides of Eq. (B3):

\footnotetext{
${ }^{4}$ See Ref. [4] Section 2.3.1; comparison with the method presented in Sections 2 and 3 of this report.

${ }^{5}$ See Ref. [6] for a formal derivation that avoids the use of logarithms.
} 


$$
\ln \left(\frac{C_{i}}{N_{i}}\right)=\sum_{j=1}^{7} a_{i j} \ln \left(B_{j}\right)
$$

Let $w_{i}=\ln \left(C_{i} / N_{i}\right)$ and $z_{i}=\ln \left(B_{i}\right)$. The set of seven equations represented by Eq. (B4) can now be written compactly in matrix form as

$$
\boldsymbol{W}=\boldsymbol{A} Z
$$

where $\boldsymbol{W}$ and $\boldsymbol{Z}$ are $1 \times 7$ arrays containing seven logarithmic elements of the form $w_{i}$ and $z_{i}$ respectively and $\boldsymbol{A}$ is the $7 \times 7$ matrix of exponents consisting of the elements $a_{i j}$.

To solve for $\boldsymbol{Z}$, multiply Eq. (B5) from the left by $\boldsymbol{A}^{-1}$ :

$$
\boldsymbol{Z}=\boldsymbol{A}^{-1} \boldsymbol{W}
$$

The existence of $\boldsymbol{A}^{-1}$ is obviously a necessary condition.

Equation (B6) represents seven individual equations. We now exponentiate each of these to eliminate the logarithms they contain. If the elements of $\boldsymbol{A}^{-1}$ are symbolized by $d_{i j}$, then the definition of the $i^{\text {th }}$ base unit is found to be:

$$
B_{i}=\prod_{j=1}^{7}\left(\frac{C_{j}}{N_{j}}\right)^{d_{i j}}
$$

which defines each base unit in terms of the defining constants and their fixed numerical values. The same procedure can be used to show that

$$
B_{i}=\prod_{j} U_{j}^{d_{i j}}
$$

is the inverse form of Eq. (B2). The definitions of the base units given in Ref. [4] can be recognized as following from Eq. (B7) when it is written with separate factors for the terms containing the $N_{j}$ and the $C_{j}$ :

$$
B_{i}=\left(\prod_{j=1}^{7} N_{j}^{-d_{i j}}\right)\left(\prod_{j=1}^{7} C_{j}^{d_{i j}}\right)
$$

Equation (B7) can also be used to define the same base units in terms of the present SI when account is taken of the different set of seven bullet points, which are shown in Appendix A.

\subsection{Comparison with the Approach Taken in Sections 2 and 3}

Sections 2 and 3 take advantage of the simplicity of Eq. (B2). The information required for each $U_{i}$ seems practically self-evident. Nevertheless, the set of equations contains all exponents $a_{i j}$ that are needed to create matrix A.

In Section 2, Table 1 is described as representing "much of the information" contained in the bullet points of Section 1. The information contained is that that the unit $U_{i}$ of the $i^{\text {th }}$ defining constant $C_{i}$ can be expressed in terms of the base units as $\mathrm{s}^{\alpha} \mathrm{m}^{\beta} \mathrm{kg}^{\gamma} \mathrm{A}^{\delta} \mathrm{K}^{\varepsilon} \mathrm{mol}^{\zeta} \mathrm{cd}^{\eta}$, where the required exponents are inferred from the $i^{\text {th }}$ bullet point of Section 1 (and, when needed, the supplementary information given below the bullet points). Table 1 thus provides an example of how the information contained in Eq. (B2) can be displayed. The important point is that Table 1 contains $\boldsymbol{A}$, and the cells of Table 1 contain the array of individual exponents $a_{i j}$ for the chosen ordering of base units and defining constants. The ordering is arbitrary from a mathematical point of view and was therefore chosen in Section 2 for didactic reasons. 


\section{Journal of Research of the National Institute of Standards and Technology}

It is also true that the exact value of the $i^{\text {th }}$ defining constant $C_{i}$ will be expressed in the revised SI in terms of the base units multiplied by an exact number. The SI value of each $C_{i}$ in the revised SI will therefore be $N_{i} \mathrm{~s}^{\alpha} \mathrm{m}^{\beta} \mathrm{kg}^{\gamma} \mathrm{A}^{\delta} \mathrm{K}^{\varepsilon} \mathrm{mol}^{\zeta} \mathrm{cd}^{\eta}$, where the exact number $N_{i}$ is stated in the $i^{\text {th }}$ bullet point of Section 1. Multiplication by a pure number does not affect the exponents.

Table 2 is designed to present a useful picture of the revised SI. With that in mind, Section 3 refers to combinations of the defining constants that have the same SI unit as the $i^{\text {th }}$ base unit $B_{i}$. The $i^{\text {th }}$ row of Table 2 is labeled with base unit $B_{i}$; the $j^{\text {th }}$ column of Table 2 is labeled with the defining constant $C_{j}$. The elements $d_{i j}$ of the table were obtained by inverting matrix $\boldsymbol{A}$, which is contained in Table 1 . Table 2 can therefore be used to find the combination of defining constants that has $B_{i}$ as its unit. The definition of each $B_{i}$ can be obtained by changing the column labels from $C_{j}$ to $C_{j} / N_{j}$ thereby making Table 2 equivalent to Eq. (B7). However, a more intuitive method is adopted for the example presented in Section 3.

\section{Acknowledgments}

The author acknowledges helpful discussions with Estefanía de Mirandés (International Bureau of Weights and Measures, BIPM), Richard J. C. Brown (National Physical Laboratory, NPL), and Paul Quincey (NPL).

\section{References}

[1] Resolution 1 of the 24th General Conference on Weights and Measures (CGPM) (2011) On the possible future revision of the International System of Units, the SI. Available at http://www.bipm.org/en/CGPM/db/24/1/

[2] Bureau International des Poids et Mesures $(2006,2014)$ The International System of Units (SI), 8th Ed. Available at https://www.bipm.org/utils/common/pdf/si_brochure_8_en.pdf

[3] Newell DB, Cabiati F, Fischer J, Fujii K, Karshenboim SG, Margolis HS, de Mirandés E, Mohr PJ, Nez F, Pachucki K, Quinn TJ, Taylor BN, Wang M, Wood BM, Zhang Z (2018) The CODATA 2017 values of h, e, k, and N A for the revision of the SI. Metrologia 55(1):L13-L16. https://doi.org/10.1088/1681-7575/aa950a

[4] Bureau International des Poids et Mesures (2018) The International System of Units (SI), draft 9th Ed., 5 Feb 2018. Available at https://www.bipm.org/utils/en/pdf/si-revised-brochure/Draft-SI-Brochure-2018.pdf. The definitive 9th edition will be available online from the BIPM on 20 May 2019.

[5] See for instance page 2 of The International System of Units (SI), Page CH, Vigoureux P, eds. National Bureau of Standards, Gaithersburg, MD, NBS Special Publication (SP) 330, 1974 edition. https://doi.org/10.6028/NBS.SP.330e1974. This English translation of the 3rd Ed. of the SI Brochure was approved by the BIPM.

[6] Mohr PJ (2008) Defining units in the quantum based SI. Metrologia 45(2):129-133. https://doi.org/10.1088/0026-1394/45/2/001

[7] Taylor BN (2018) Quantity calculus, fundamental Constants, and SI Units. Journal of Research of the National Institute of Standards and Technology 123:123008. https://doi.org/10.6028/jres.123.008

[8] Bureau International des Poids et Mesures (2018) Future Revision of the SI Brand Book, p 18. Available at https://www.bipm.org/utils/common/pdf/SI-Brand-Book.pdf

[9] Wikipedia (2018) Proposed redefinition of SI base units. Available at https://en.wikipedia.org/wiki/Proposed_redefinition_of_SI_base_units

[10] Taylor BN (2011) The current SI seen from the perspective of the proposed new SI. Journal of Research of the National Institute of Standards and Technology 116(6):797-807. https://doi.org/10.6028/jres.116.022

[11] See address by CIPM President André Danjon (1960) Proceedings of the 11th CGPM, pp 23-25 [in French]. Available at http://www.bipm.org/utils/common/pdf/CGPM/CGPM11.pdf

[12] CIPM Consultative Committees (2017) Joint statement on the proposed revision of the SI. Available at https://www.bipm.org/utils/common/pdf/SI-statement.pdf 
About the author: Richard S. Davis was a National Research Council Postdoctoral Associate in the Electricity Division at the National Bureau of Standards (now NIST) in 1974-1976 and then a member of the technical staff. In 1990 he joined the International Bureau of Weights and Measures (BIPM). During his career, he helped to measure three fundamental constants: the Faraday constant, the molar gas constant, and the gravitational constant. (The values of the first two of these are defined exactly by the new definitions of the ampere, mole, and kelvin in the revised SI, and so cannot be measured again.) From 1993 until his retirement in 2010, he was head of the Mass Department of the BIPM, where the International Prototype of the Kilogram was used and is now conserved.

This article was adapted from BIPM publication Rapport BIPM-2018/02.

The National Institute of Standards and Technology is an agency of the U.S. Department of Commerce. 